\title{
Status of NA62
}

\author{
Cristina Lazzeroni* ${ }^{* \dagger}$ \\ University of Birmingham, $U K$ \\ E-mail: cristina.lazzeroni@cern.ch
}

The NA62 experiment at CERN aims to search for new phenomena beyond the Standard Model, by measuring two of the theoretically cleanest kaon decays. Two phases can be distinguished in the NA62 physics programme. During the current short term phase (data taking completed in years 2007-8), the ratio $R_{K}=\Gamma\left(K^{+} \rightarrow e v_{e}(\gamma)\right) / \Gamma\left(K^{+} \rightarrow \mu v_{\mu}(\gamma)\right)$ of leptonic decay rates is studied, which tests the structure of weak interactions and lepton flavour universality. In the second phase (long term) the experiment will focus on the measurement of the branching ratio of the very rare kaon decay $K^{+} \rightarrow \pi^{+} v \bar{v}$ with a $10 \%$ precision. In this paper, firstly the $R_{K}$ analysis is summarized, and the preliminary result $R_{K}=$ based on $40 \%$ of data collected in 2007 is discussed. Then a status report of the long term project is presented, including a discussion of the beam line, detectors, and the experimental strategy.

Flavor Physics and CP Violation - FPCP 2010

May 25-29, 2010

Turin, Italy

* Speaker.

${ }^{\dagger}$ A footnote may follow. 


\section{Kaon leptonic decays and $R_{K}$}

Due to the V-A structure of the weak interactions, the Standard Model (SM) rates of the leptonic meson decays $P^{+} \rightarrow l^{+} v$ are helicity suppressed. Within the two-Higgs doublet models (2HDM), the charged Higgs boson exchange induces a tree-level contribution, proportional to the Yukawa couplings of quarks and leptons[1], and such contribution can compete with the $W$ exchange, thanks to the above suppression. At tree level, the $H^{ \pm}$exchange contribution to $P^{+} \rightarrow l^{+} v$ decay widths (with $P=\pi, K, B$ ) is lepton flavour independent, and is approximately described by[2]

$$
\frac{\Delta \gamma\left(P^{+} \rightarrow l^{+} v\right)}{\Gamma^{S M}\left(P^{+} \rightarrow l^{+} v\right)}=\approx-2\left(\frac{M_{P}}{M_{H}}\right)^{2} \frac{\tan ^{2} \beta}{1+\varepsilon^{0} \tan \beta}
$$

where $M_{H}$ is the charged Higgs boson mass, $\tan \beta$ is the ratio of vacuum expectation values of the two Higgs doublets, and $\varepsilon^{0} \sim 10^{-2}$ is an effective coupling. For $\tan \beta=40, M_{H}=500 \mathrm{GeV} / \mathrm{c}^{2}$ ) $30 \%$ relative suppression of $B^{+}$decays, and $0.3 \%$ suppression of $K^{+}$decays is predicted. However the searches for new physics in these decay rates are hindered by the uncertainties of the SM predictions. On the contrary, the ratio of kaon leptonic decay rates $R_{K}=\Gamma\left(K^{+} \rightarrow e^{+} v_{e}\right) / \Gamma\left(K^{+} \rightarrow\right.$ $\mu^{+} v_{\mu}$ ) has been calculated with an excellent accuracy within the SM[3]:

$$
R_{K}^{S M}=\left(m_{e} / m_{\mu}\right)^{2}\left(\frac{m_{K}^{2}-m_{e}^{2}}{m_{K}^{2}-m_{\mu}^{2}}\right)^{2}\left(1+\delta R_{Q E D}\right)=(2.477 \pm 0.001) \times 10^{-5}
$$

where $\delta R_{Q E D}=(-3.78 \pm 0.04) \%$ is a correction due to the inner bremsstrahlung (IB) radiative process. The ratio $R_{K}$ is sensitive to lepton flavour universality violation (LFV) effects originating at one-loop level from $H^{ \pm}$exchange in $2 \operatorname{HDM}[4,5]$, and the mixing effects in the right-handed slepton sector, providing a unique probe into this aspect of supersymmetric flavour physics[6]. $R_{K}$ receives the following leading-order contribution due to LFV coupling of the Higgs boson:

$$
\frac{\Delta R_{K}}{R_{K}^{S M}}=\left(M_{K} / M_{H}\right)^{4}\left(M_{\tau} / M_{e}\right)^{2}\left|\Delta_{R}^{31}\right|^{2} \tan ^{6} \beta
$$

where $\Delta_{R}^{31} \sim 10^{-3}$ is the mixing parameter between the superpartners of the right-handed leptons. This can enhance $R_{K}$ by $\mathrm{O}(1 \%)$. The current world average (including only final results, and thus ignoring the preliminary NA48/2 ones) is $R_{K}^{W A}=(2.490 \pm 0.030) \times 10^{-5}$, dominated by a recent measurement by the KLOE collaboration[7]. The NA62 experiment at CERN collected a dedicated data sample in $2007 Đ 08$, aiming at a measurement of $R_{K}$ with a $0.4 \%$ precision.

\subsection{Beam, detector and data taking}

The beam line and setup of the NA48/2 experiment[8] were used for the NA62 2007Đ08 data taking. Experimental conditions and trigger logic were optimized for the $R_{K}$ measurement. The beam line delivered simultaneous unseparated $\mathrm{K}^{+}$and $\mathrm{K}^{-}$beams derived from $400 \mathrm{GeV} / \mathrm{c}$ primary protons extracted from the CERN SPS. Most of the data, including the sample used for the present analysis, were collected with the $K^{+}$beam only, as the muon sweeping system provided better suppression of the positive beam halo component. A narrow momentum band of $(74.0 \pm 1.6) \mathrm{GeV} / \mathrm{c}$ was used to minimize the corresponding contribution to resolution in kinematical variables. The fiducial decay region was contained in a $114 \mathrm{~m}$ long cylindrical vacuum tank. The beam flux at the 
entrance to the decay volume was $2.5 \times 10^{7}$ particles per pulse of $4.8 \mathrm{~s}$ duration. The fractions of $K^{+}, \pi^{+}, p, e^{+}, \mu^{+}$in the beam were $0.05,0.63,0.21,0.10,0.01$ respectively. The fraction of beam kaons decaying in the vacuum tank at nominal momentum was $18 \%$. The transverse size of the beam within the decay volume was $x=y=7 \mathrm{~mm}(\mathrm{rms})$, and its angular divergence was negligible. Among the subdetectors located downstream the decay volume, a magnetic spectrometer, a plastic scintillator hodoscope (HOD) and a liquid krypton electromagnetic calorimeter ( $\mathrm{LKr}$ ) were principal for the measurement. The spectrometer, used to detect charged products of kaon decays, was composed of four drift chambers (DCHs) and a dipole magnet. The HOD consisted of two planes of strip-shaped counters and produced fast trigger signals. The LKr, used for particle identification and as a veto, was an almost homogeneous ionization chamber, $27 X^{0}$ deep, segmented transversally into 13,248 cells ( $2 \times 2 \mathrm{~cm}^{2}$ each), and with no longitudinal segmentation. A beam pipe traversing the centres of the detectors allowed undecayed beam particles and muons from decays of beam pions to continue their path in vacuum. A minimum bias trigger configuration was employed, resulting in high efficiency with relatively low purity. The Ke2 trigger condition consisted of coincidence of hits in the HOD planes (Q1) with $10 \mathrm{GeV} \mathrm{LKr}$ energy deposition. The $K \mu 2$ trigger condition consisted of the Q1 signal alone downscaled by a factor of 150. Loose lower and upper limits on DCH activity were also applied. The main data taking took place during four months starting in June 2007. Two additional weeks of data taking allocated in September 2008 were used to collect special data samples for studies of systematic effects. The present analysis is based on $40 \%$ of the data sample.

\subsection{Analysis strategy and event selection}

The analysis strategy is based on counting the numbers of reconstructed $K e 2$ and $K \mu 2$ candidates collected at the same time. Consequently the result does not rely on kaon flux measurement, and several systematic effects cancel to first order. To take into account the significant dependence of signal acceptance and background level on lepton momentum, the measurement is performed independently in bins of this observable: 10 bins covering a lepton momentum range of $[15 ; 65] \mathrm{GeV} / \mathrm{c}$ are used. The ratio $R_{K}$ in each bin is computed as:

$$
R_{K}=\frac{1}{D} \cdot \frac{N\left(K_{e 2}\right)-N_{B}\left(K_{e 2}\right)}{N\left(K_{\mu 2}\right)-N_{B}\left(K_{\mu 2}\right)} \cdot \frac{A\left(K_{\mu 2}\right)}{A\left(K_{e 2}\right)} \cdot \frac{f_{\mu} \times \varepsilon\left(K_{\mu 2}\right)}{f_{e} \times \varepsilon\left(K_{e 2}\right)} \cdot \frac{1}{f_{l k r}}
$$

where $\mathrm{N}$ are the numbers of selected signal candidates, $N_{B}$ are numbers of background events, $A$ is the geometric acceptance correction, $f$ are efficiencies of lepton identification, $\varepsilon$ are trigger efficiencies, $f_{L K r}$ is the global efficiency of the $\mathrm{LKr}$ readout, and $D=150$ is the downscaling factor of the $K \mu 2$ trigger.

A detailed Monte Carlo (MC) simulation including beam line optics, full detector geometry and material description, stray magnetic fields, local inefficiencies of $\mathrm{DCH}$ wires, and time variations of the above throughout the running period, was used to evaluate the acceptance correction and the geometric parts of the acceptances for background processes. The radiative processes are simulated in one-photon approximation[3]; the resummation of leading logarithms [9] is neglected at this stage. Simulations are used to a limited extent only: particle identification, trigger and readout efficiencies are measured directly using data. 
Due to the topological similarity of $K e 2$ and $K \mu 2$ decays, a large part of the selection conditions is common for both decays: (1) exactly one reconstructed particle of positive electric charge; (2) its momentum must be $15 \mathrm{GeV} / c<p<65 \mathrm{GeV} / c$ (the lower limit is due to the $10 \mathrm{GeV} \mathrm{LKr}$ energy deposit trigger requirement in $K e 2$ trigger); (3) extrapolated track impact points in $\mathrm{DCH}$, $\mathrm{LKr}$ and HOD are within their geometrical acceptances; (4) no LKr energy deposition clusters with energy $E>2 \mathrm{GeV}$ and not associated to the track, to suppress background from other kaon decays; (5) distance between the charged track and the nominal kaon beam axis $C D A<3 \mathrm{~cm}$, (6) decay vertex longitudinal position within the nominal decay volume (the latter condition is optimized in each lepton momentum bin). The following two principal selection criteria are different for the $K e 2$ and $K \mu 2$ decays: (1) the kinematic identification is based on the reconstructed squared missing mass assuming the track to be a positron or a muon; (2) particle identification is based on the ratio $E / p$ of track energy deposit in the $\mathrm{LKr}$ calorimeter to its momentum measured by the spectrometer. The quantity $M_{\text {miss }}^{2}=\left(P_{K}-P_{l}\right)^{2}$ is defined, where $P_{K}$ and $P_{l}(l=e, \mu)$ are the four-momenta of the kaon (average beam momentum assumed) and the lepton (positron or muon mass assumed). A selection condition $\left|M_{\text {miss }}^{2}(e, \mu)\right|<M_{0}^{2}$ is applied, where $M_{0}^{2}$ varies from 0.009 to $0.013\left(\mathrm{GeV} / \mathrm{c}^{2}\right)^{2}$ with lepton momentum bins depending on $M_{\text {miss }}^{2}$ resolution. Particles with $0.95<E / p<1.1$ (or $E / p<0.85$ ) are identified as positrons (or muons).

\subsection{Background rejection}

$K \mu 2$ decays with a mis-identified muon are the main background source in the $K e 2$ sample. Sufficient kinematic separation between electron and muon decays is not achievable at high lepton momentum $(p>30 \mathrm{GeV} / c)$, as shown in Fig.1(Left). The probability of muon identification as positron in that momentum range is $P(\mu \rightarrow e) \sim 4 \times 10^{-6}$, since $E / p>0.95$ due to catastrophic bremsstrahlung in or in front of the LKr. Since such probability is non-negligible for the measurement in question, a direct measurement of $P(\mu \rightarrow e)$ to $10^{-2}$ relative precision is necessary to validate the theoretical calculation of the bremsstrahlung cross-section[10] in the high energy $\gamma$ range used to evaluate the $K \mu 2$ background. The available muon samples are typically affected by $\sim 10^{-4}$ electron/positron contamination due to $\mu \rightarrow e$ decays in flight, which obstructs the probability measurements. In order to obtain sufficiently pure muon samples, a $9.2 X^{0}$ thick lead $(\mathrm{Pb})$ wall covering $20 \%$ of the geometric acceptance was installed in front of the $\mathrm{LKr}$ calorimeter (between the two HOD planes) during a fraction of the data taking. In the samples of tracks traversing the $\mathrm{Pb}$ and having $E / p>0.95$, the electron component is suppressed to a level of $\sim 10^{-7}$ by energy losses in $\mathrm{Pb}$. The momentum dependence of $P(\mu \rightarrow e)$ for muons traversing the $\mathrm{Pb}$ has been measured with a data sample collected during a special muon run of $20 \mathrm{~h}$ duration, and compared to the results of a dedicated Geant4-based MC simulation of the region downstream the spectrometer involving standard energy loss processes and bremsstrahlung[10]. The data/MC comparison, seen in Fig.1(Right), shows good agreement in a wide momentum range within statistical errors, which validates the cross-section calculation at the corresponding precision level. The simulation shows that the $\mathrm{Pb}$ wall modifies $P(\mu \rightarrow e)$ via two principal mechanisms: 1) muon energy loss in the $\mathrm{Pb}$ by ionization, dominating at low momentum; 2) bremsstrahlung in $\mathrm{Pb}$, dominating at high momentum. To estimate the $K \mu 2$ background contamination, the kinematic suppression factor is computed with the standard setup simulation, while the validated simulation of muon interaction 
in the $\mathrm{LKr}$ is employed to account for the $P(\mu \rightarrow e)$ suppression. Uncertainty of the background estimate is due to the limited size of the data sample used to validate the simulation.
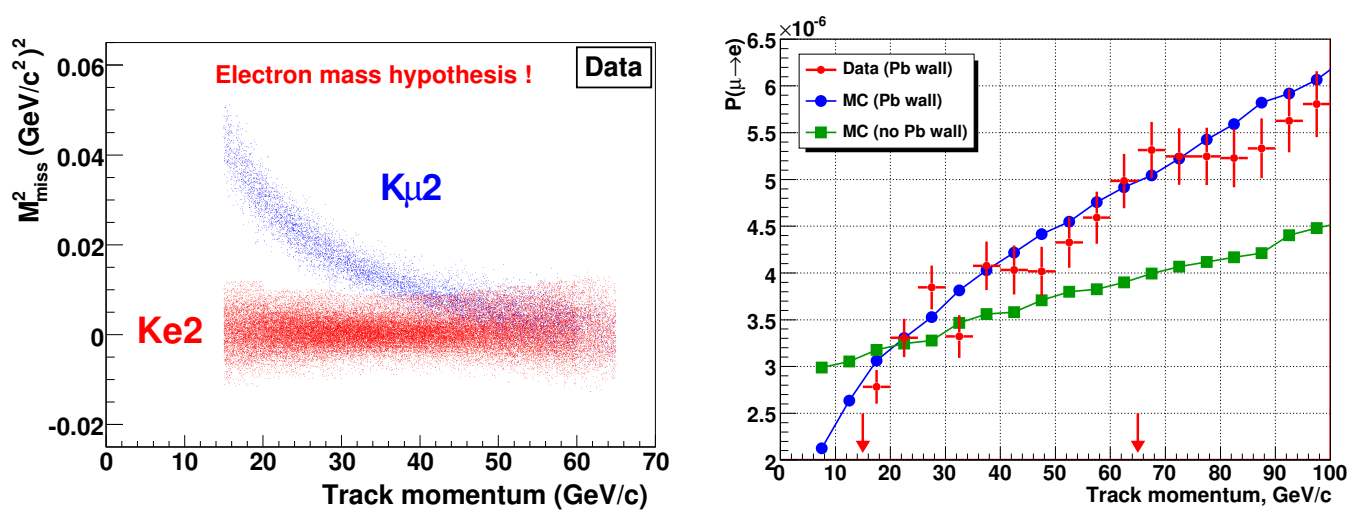

Figure 1: (Left) Missing mass squared in positron hypothesis vs lepton momentum for reconstructed $K e 2$ and $K \mu 2$ decays: kinematic separation is possible at low lepton momentum only. (Right) Measured and simulated probability of muon identification as electron/positron vs its momentum: data with the $\mathrm{Pb}$ wall, MC simulations with and without the $\mathrm{Pb}$ wall (the signal region is marked with arrows).

$K \mu 2$ decay followed by $\mu \rightarrow e$ decay could contribute significantly to the background, but energetic forward daughter positrons compatible to $K e 2$ topology are suppressed due to muon polarization[11].

The structure dependent (SD) component of the radiative decay $K \rightarrow e v \gamma$ is an reducible background to $R_{K}$, and has a rate similar to that of $K e 2$. Theoretical rate calculations depend on the form factor model, and have a similar precision. Energetic positrons $\left(E(e)>230 \mathrm{MeV}\right.$ in $\mathrm{K}^{+}$frame) escaping detector acceptance contribute to the background, which estimate using MC has a $15 \%$ uncertainty due to limited knowledge of the process. A recent measurement by KLOE[7] is not used here since published after announcement of the NA62 preliminary result.

Beam halo background in the $K e 2$ sample induced by halo muons (undergoing decay in flight or mis-identified) is measured directly by reconstructing $K_{e 2}^{+}$candidates from a control $K^{-}$data sample collected with the $K^{+}$beam dumped. Background rate and kinematical distribution are qualitatively reproduced by a halo simulation. The uncertainty is due to the limited size of the $K^{-}$ sample. Beam halo is the only significant background source in the $K \mu 2$ sample, measured to be $0.25 \%$ (with a negligible uncertainty) using the same technique as for $K e 2$ decays.

The number of candidates is $N(K e 2)=51089$ (about four times the statistics collected by KLOE) and $N(K \mu 2)=15.56 \times 10^{6}$. The missing mass distributions (electron hypothesis) of data events and backgrounds are presented in Fig.2(Left). Backgrounds integrated over lepton momentum are summarized in Table1; their distributions over lepton momentum are presented in Fig.2(Right). The total background is $(8.03 \pm 0.23) \%$.

\subsection{Systematic uncertainties and result}

Positron identification efficiency is measured directly as a function of momentum and $\mathrm{LKr}$ impact point, using pure samples of electrons and positrons obtained by kinematic selection of $K^{+} \rightarrow \pi^{0} e^{+} v$ decays collected concurrently with the $K e 2$ sample, and $K_{L}^{0} \rightarrow \pi^{ \pm} e^{ \pm} v$ decays from 

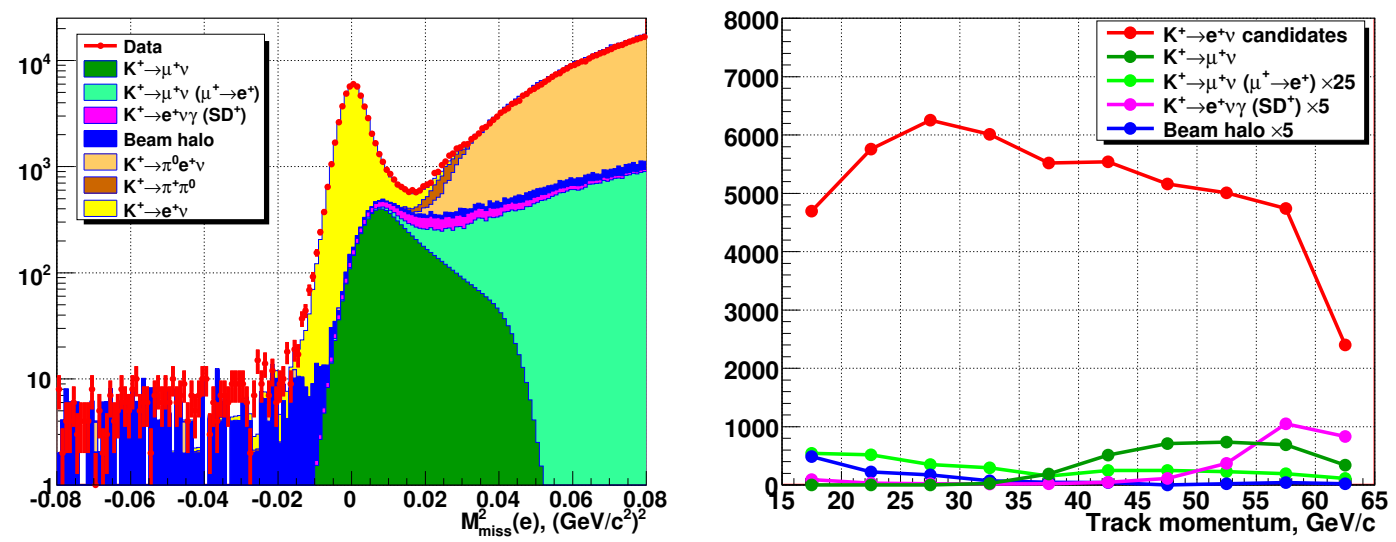

Figure 2: (Left) Reconstructed squared missing mass distribution for the $K e 2$ candidates: data (dots) presented as sum of MC signal and background contributions (filled areas). (Right) Numbers of $K e 2$ candidates and background events in lepton momentum bins.

Table 1: Summary of background sources in $K e 2$ sample.

\begin{tabular}{|cc|cc|cc|}
\hline Source & $N_{B} / N_{\text {tot }}(\%)$ & Source & $N_{B} / N_{\text {tot }}(\%)$ & Source & $N_{B} / N_{\text {tot }}(\%)$ \\
\hline$K \mu 2$ & $6.28 \pm 0.17$ & $K e 2 \gamma(\mathrm{SD})$ & $1.02 \pm 0.15$ & $K e 3$ & 0.03 \\
$K \mu 2(\mu \rightarrow e)$ & $0.23 \pm 0.01$ & Beam halo & $1.45 \pm 0.04$ & $K 2 \pi$ & 0.03 \\
\hline
\end{tabular}

a special $K_{L}^{0}$ run. The $K^{+}$and $K_{L}^{0}$ measurements are in good agreement. The measured $f_{e}$ averaged over the Ke2 sample is $(99.20 \pm 0.05) \%$. Muon identification inefficiency is negligible. The geometric acceptance correction is strongly affected by the radiative $K e 2$ (IB) decays. A conservative systematic uncertainty is attributed to approximations used in the $K e 2$ (IB) simulation. The resummation of leading logarithms[9] is not taken into account; however no systematic error is ascribed to that. An additional systematic uncertainty reflects the precision of beam line and apparatus description in the MC simulation. Trigger efficiency correction $\varepsilon=99.9 \%$ accounts for the fact that the condition $E_{L K r}>10 \mathrm{GeV}$ is used in the $K e 2$ trigger only. A conservative systematic uncertainty of $0.3 \%$ is ascribed due to effects of trigger dead time which affect the two modes differently. $\mathrm{LKr}$ global readout efficiency $f_{L K r}$ is measured directly to be $(99.80 \pm 0.01) \%$ and is checked to be stable in time using an independent $\mathrm{LKr}$ readout system.

The independent measurements of $R_{K}$ in lepton momentum bins, and the result combined over the momentum bins are presented in Fig.3(Left). The uncertainties are summarised in Table2. The preliminary result, based on $40 \%$ of data, is $R_{K}=\left(2.500 \pm 0.012_{\text {stat. }} \pm 0.011_{\text {syst. }}\right) \times 10^{-5}=$ $(2.500 \pm 0.016) \times 10^{-5}$, consistent with the SM expectation. Analysis of the whole $2007 \mathrm{D} 08$ data sample is expected to decrease the uncertainty down to $0.4 \%$. A summary of available $R_{K}$ measurements is presented in Fig.3(Right): the current world average is $(2.498 \pm 0.014) \times 10^{-5}$.

\section{The $K^{+} \rightarrow p i^{+} v \bar{v}$ measurement}

The $B R\left(K^{+} \rightarrow p i^{+} v \bar{v}\right)$ is predicted within the Standard Model Theory to be $(0.85 \pm 0.07) \times$ 

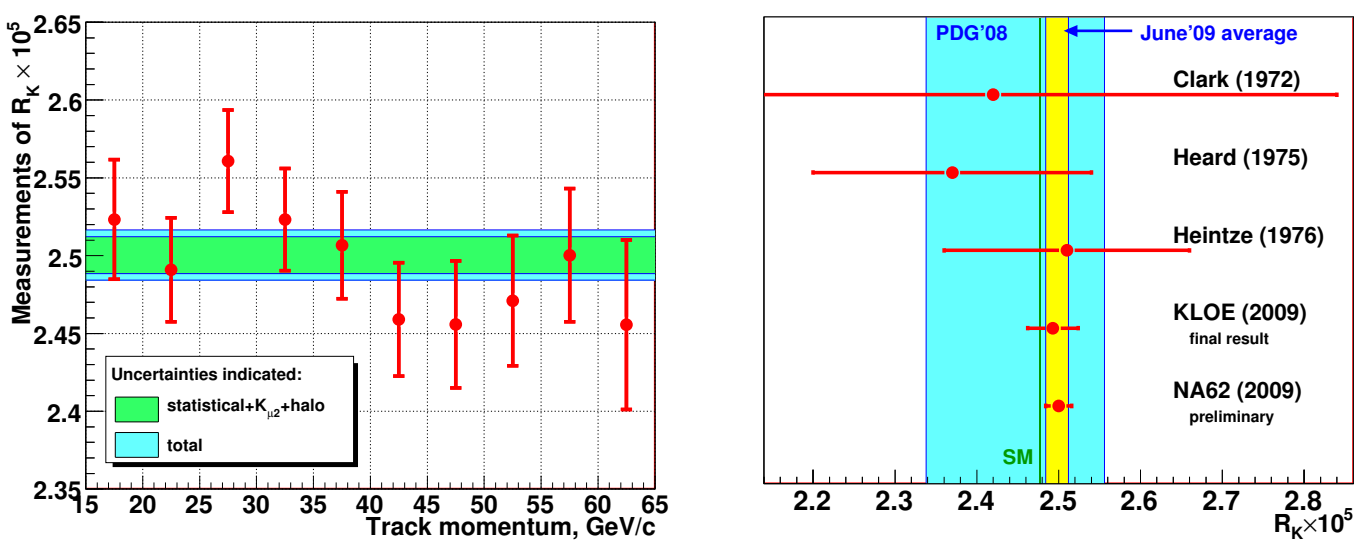

Figure 3: (Left) Measurements of $R_{K}$ in lepton momentum bins. (Right) Various measurements of $R_{K}$ and their world average.

Table 2: Summary of statistical and systematic uncertainties on $R_{K}$.

\begin{tabular}{|cc|cc|cc|}
\hline Source & $\delta R_{K} \times 10^{5}$ & Source & $\delta R_{K} \times 10^{5}$ & Source & $\delta R_{K} \times 10^{5}$ \\
\hline Statistical & 0.012 & Beam halo & 0.001 & Geom. accep. & 0.002 \\
$K \mu 2$ & 0.004 & Positron ID & 0.001 & Trigger dead time & 0.07 \\
$K e 2 \gamma(\mathrm{SD})$ & 0.004 & IB simulation & 0.007 & & \\
\hline
\end{tabular}

$10^{-10}$ with a small error due to isospin symmetry. Therefore a $10 \%$ measurement represents a powerful probe of possible physics beyond the Standard Model. The NA62 experiment at CERN[12] will collect about 100 signal events every 2 years of data taking starting in 2013, with a maximum of $10 \%$ background.

\subsection{The beam}

Assuming a signal acceptance of the order of $10 \%, 10^{13} \mathrm{~K}^{+}$will be needed, and will be provided by the existing CERN accelerator system. The NA62 experiment will make use of the experimental cavern once dedicated to NA48. The SPS will deliver $400 \mathrm{GeV} / \mathrm{c}$ momentum protons with the slow extraction method in pulses of $3 \times 10^{12}$ protons in $4.8 \mathrm{~s}$ with a duty cycle of 16.8 s. Primary protons will impinge on a beryllium target forming a secondary beam which will be selected to have $75 \mathrm{GeV} / \mathrm{c}$ momentum with $1 \%$ dispersion. This secondary beam will contain about $6 \%$ of $K^{+}$, therefore providing $4.5 \times 10^{12} K^{+}$decays per year over the $60 \mathrm{~m}$ long fiducial volume of the experiment.

Pions and protons in the beam can mimic a kaon decay only if they undergo beam-gas interactions. The decay volume will be evacuated at $10^{-6}$ mbar and $K^{+}$will be positively identified by a Cherenkov detector (CEDAR) in the beam line, filled with 4 bar Hydrogen. The CEDAR pressure and optical diaphragm will be tuned to select only $\mathrm{K}^{+}$with a minimal contamination, resulting in a rate of $45 \mathrm{MHz}$. 
All beam particles will pass through an achromat magnetic system where three silicon pixel stations will be located to measure the particle momentum and direction. This detector, called Gigatracker (GTK), will sustain an instantaneous rate of $750 \mathrm{MHz}$. Each GTK station will have $18000300 \times 300 \mu \mathrm{m}^{2}$ pixels, and will correspond to less than $0.5 \%$ radiation length. The expected performances are $0.2 \%$ uncertainty of the momentum, $17 \mu \mathrm{m}$ angular resolution and $150 \mathrm{ps}$ time resolution.

Particles produced by beam interactions in the last GTK station and in the final beam collimator will be vetoed by a charged anti-counter (CHANTI) arranged in 6 stations located downstream of the GTK.

\subsection{Kinematics}

The signal is composed by an incoming charged kaon and an outgoing charged pion of the same charge, and nothing else. The squared missing mass, $m_{m i s s}^{2}$, is defined as the squared difference of $K^{+}$and $\pi^{+}$four-momenta, and is used to select the events. Considering all known decay modes, $92 \%$ of the $K^{+}$decays have $m_{\text {miss }}^{2}$ between the negatively reconstructed $K^{+} \rightarrow \mu^{+} v$ distribution and the $K^{+} \rightarrow \pi^{+} \pi^{0}$ peak (Region I: $0<m_{\text {miss }}^{2}<0.01 \mathrm{GeV}^{2} / \mathrm{c}^{4}$ ), and between this peak and the beginning of the three pion decay distribution (Region II: $0.026<m_{\text {miss }}^{2}<0.068 \mathrm{GeV}^{2} / \mathrm{c}^{4}$ ).

The missing mass is reconstructed using the information from GTK for $\mathrm{K}^{+}$and a downstream magnetic spectrometer for $\pi^{+}$. The spectrometer is formed by 4 straw tubes chambers, with 4 views per chamber and 4 layers per view, and it operates in vacuum. Each view corresponds to $0.1 \%$ radiation length. A track resolution of $130 \mu \mathrm{m}$ per view was measured on a prototype during a test beam in 2007. A dipole magnet with $256 \mathrm{MeV} / \mathrm{c} p_{t}$ kick will be placed between the second and the third chamber. A momentum resolution of $0.3 \% \pm 0.007 \% \times P_{\pi}$ is predicted, where $P_{\pi}$ is the pion momentum in $\mathrm{GeV}$. The region where undecayed beam particles will pass, will not be instrumented with straws. With the available simulation, a $m_{\text {miss }}^{2}$ resolution of $10^{-3} \mathrm{GeV}^{2} / \mathrm{c}^{4}$ is expected, dominated by the angle between $K^{+}$and $\pi^{+}$.

\subsection{Backgrounds}

According to simulation, the cut on $m_{\text {miss }}^{2}$ will give a suppression factor of $0.5 \times 10^{-5}$ for the most probable $K^{+}$decay, $K^{+} \rightarrow \mu^{+} v$. A further suppression of $10^{-5}$ will be achieved using the different penetrating power between muons and pions. A muon veto (MUV) will be used for this purpose. The first of its modules is the front module of the NA48 hadron calorimeter[8], while the second module will be built using a sandwich of iron plates and scintillator stripes. A fast scintillator plane (MUV3) will be located downstream, to veto most of the $10 \mathrm{MHz}$ muon rate at trigger level. A further $10^{-2}$ suppression will be achieved by discriminating pions from muons using a Cherenkov detector (RICH) filled with Neon at atmospheric pressure, selecting a momentum fiducial region between 15 and $35 \mathrm{GeV} / \mathrm{c}$. A beam pipe will host the undecayed particles. The Cherenkov light will be detected by single anode photomultipliers, located in two spots on the left and on the right of the beam pipe, to avoid the beam pipe shadow. A muon suppression factor of $0.7 \%$ has been measured during a test beam in 2009 , together with an event time resolution of $70 \mathrm{ps}$. A scintillator charged hodoscope (CHOD) will be located downstream of the RICH and will be used to veto inelastic interactions in the previous detectors and also for triggering purposes. 
According to simulation, the cut on $m_{\text {miss }}^{2}$ will give a suppression factor of $5 \times 10^{-5}$ for the second most probable $K^{+}$decay, $K^{+} \rightarrow \pi^{+} \pi^{0}$. A further $10^{-8}$ suppression will come by vetoing at least one of the photons from $\pi^{0}$ decays. This will be achieved by a combination of 3 different detectors, covering a different angular acceptance: the region between 8.5 and $50 \mathrm{mrad}$ will be covered by the large angle veto (LAV); the region between 1 and $8.5 \mathrm{mrad}$ by the electromagnetic calorimeter (LKR); and finally below $1 \mathrm{mrad}$ by the inner ring calorimeter (IRC) and the small angle calorimeter (SAC). The LAV will be made of lead crystals recovered from the OPAL experiment, and arranged in 12 rings along the experimental region. All the rings will be in vacuum except the last one. According to results from various test beams, photons with energy larger than $1 \mathrm{GeV}$ will be vetoed with $10^{-5}$ inefficiency, degrading gradually to $10^{-4}$ for energies below 1 $\mathrm{GeV}$. The LKR, an homogenous calorimeter filled with liquid krypton, was used already by the NA48 experiment[8], and will veto photon with energy above $5 \mathrm{GeV}$ with an inefficiency of $10^{-5}$, degrading gradually to $10^{-4}$ for energies between 1 and $5 \mathrm{GeV}$. The IRC is a shaslyk calorimeter shaped as a ring and placed around the beam pipe, downstream of the RICH. It will veto photons directed to passive area of the LRK at small angle. The SAC, also a shaslyk calorimeter, will be placed at the very end of the experimental apparatus, downstream of the muon vetoes. A dipole magnet, placed in front of the SAC, will deviate beam charged particle so that only photons can reach the SAC.

About $92 \%$ of the background is kinematically constrained (two-bodies decays, see above) and can be rejected with a selection on $m_{m i s s}^{2}$. The residual $8 \%$ must be rejected by vetoes and particle identification. The required muon rejection is achieved by exploiting the different penetration of muons and pions through matter $\left(\sim 10^{5}\right)$, in addition to the RICH suppression $\left(\sim 10^{3}\right)$. The required photon rejection $\left(\sim 10^{8}\right)$ is achieved by selecting pion with $p<35 \mathrm{GeV} / \mathrm{c}$, so that for background from $\pi \pi^{0}$ decays at least $40 \mathrm{GeV}$ of energy is deposited in hermetic calorimeters and the $\pi^{0}$ can hardly be missed.

Tight coincidences between the GTK track time and the RICH track time will be used to reject an accidental matching between a pion in the downstream spectrometer with an non-kaon beam particle. The trigger signal for a charged track will be provided by the RICH and the CHOD; muon vetoing will be made by MUV3 while photon vetoing will be achieved by LAV and LKR, reducing the trigger rate down to $1 \mathrm{MHz}$. A dedicated $\mathrm{PC}$ farm, analyzing data online, will further reduce the data collection by a factor 40 . The signal acceptance is expected to be $3.5 \%$ in Region I and $10.9 \%$ in Region II, allowing to record 55 events in 100 days of running at $50 \%$ efficiency. The total background level is predicted to be around $10 \%$.

\section{Conclusions}

Kaon decays continue to provide valuable information to the physics of weak and strong interactions. Rare decays offer unique possibility to study contribution from New Physics processes with high sensitivity. High precision experiments are complementary to the high energy frontier. Once the New Physics is discovered at LHC, flavour physics will play a major role to disentangle among the different possible New Physics scenarios.

Lepton flavour universality has been tested by NA62 via the $R_{K}=\Gamma\left(K^{+} \rightarrow e v_{e}(\gamma)\right) / \Gamma\left(K^{+} \rightarrow\right.$ $\left.\mu v_{\mu}(\gamma)\right)=\left(2.500 \pm 0.012_{\text {stat. }} \pm 0.011_{\text {syst. }}\right) \times 10^{-5}=(2.500 \pm 0.016) \times 10^{-5}$, using $40 \%$ of data 
collected in 2007. The result is consistent with Standard Model expectations.

The ultra-rare $K^{+} \rightarrow \pi^{+} v v$ decay is an unique environment to search for new physics. The NA62 experiment at CERN-SPS aims to collect O(100) events with $10 \%$ background, in 2 years of data taking starting in 2013.

\section{References}

[1] W.S. Hou, Phys. Rev. D48 (1993) 2342.

[2] G. Isidori and P. Paradisi, Phys. Lett. B639 (2006) 499.

[3] V. Cirigliano and I. Rosell, Phys. Rev. Lett. 99 (2007) 231801.

[4] A. Masiero, P. Paradisi and R. Petronzio, Phys. Rev. D74 (2006) 011701.

[5] A. Masiero, P. Paradisi and R. Petronzio, JHEP 0811 (2008) 42.

[6] J. Ellis, S. Lola and M. Raidal, Nucl. Phys. B812 (2009) 128.

[7] F. Ambrosino et al., Eur. Phys. J. C64 (2009) 627. Erratum-ibid. C65 (2010) 703.

[8] V.Fanti et al., The beam and detector for the NA48 neutral kaon CP violation experiment at CERN, NIM A574 (2007) 433.

[9] C. Gatti, Eur. Phys. J. C45 (2006) 417.

[10] S.R. Kelner, R.P. Kokoulin and A.A. Petrukhin, Phys. Atom. Nucl. 60 (1997) 576.

[11] L. Michel, Proc. Phys. Soc. A63 (1950) 514.

[12] G.Anelli et al., Proposal to measure the rare decay $K^{+} \rightarrow p i^{+} v \bar{v}$ at the CERN SPS, CERN-SPSC-2005-013 and CERN-SPSC-P326 (2005). 